\title{
Effect of insulin resistance on whole blood mRNA and microRNA expression affecting bone turnover
}

\author{
Urszula Razny, Anna Polus, Joanna Goralska, Anna Zdzienicka, Anna Gruca, \\ Maria Kapusta, Maria Biela, Aldona Dembinska-Kiec, Bogdan Solnica and \\ Malgorzata Malczewska-Malec
}

Correspondence should be addressed to U Razny

Department of Clinical Biochemistry, Jagiellonian University Medical College, Krakow, Poland

Email

urazny@cm-uj.krakow.pl

\begin{abstract}
Objective: To evaluate the effect of insulin resistance in obesity on the expression in whole blood of mRNA and miRNA affecting bone homeostasis as well as to estimate the influence of oral glucose load (OGTT) on serum osteocalcin concentration in obese individuals with and without insulin resistance.

Design: Cross-sectional study.

Methods: Carboxylated (cOC), undercarboxylated (ucOC) and total osteocalcin were measured by ELISA in the serum of obese subjects with insulin resistance $(n=41)$ and obese subjects without insulin resistance $(n=41)($ control group) during OGTT. Analysis of gene expression (microarray) and miRNAs (real-time PCR) was performed in venous blood (representating samples) collected before OGTT from obese with insulin resistance and controls.

Results: Obese subjects with insulin resistance (higher HOMA-IR and lower oral glucose insulin sensitivity index) presented significantly increased expression of WNT signalling inhibitors (DKK1, DKK2, SOST, SFRP1) and downregulation of the key factor in WNT signalling - $\beta$ catenin participating in osteoblasts differentiation. Expression of miRNA involved in osteoblastogenesis was also inhibited (miR-29b, miR-181a, miR-210, miR-324-3p). During OGTT, contrary to the control group, subjects with insulin resistance presented suppression of cOC and total OC decrease after 1 and 2 h of oral glucose load.

Conclusions: Obese subjects with insulin resistance may have defects in osteoblastogenesis that was demonstrated via key signalling molecules mRNA downregulation, and increased expression of WNT antagonists as well as inhibition of expression of miRNA participating in the regulation of osteoblast differentiation. Disturbed osteoblastogenesis in insulin-resistant subjects results in the suppression of blood carboxylated and total osteocalcin decrease during OGTT.
\end{abstract}

European Journal of Endocrinology

(2019) 181, 525-537

\section{Introduction}

Inappropriate secretion of insulin and insulin resistance as well as chronic low-grade inflammation in obesity leads to disturbances in bone turnover. Ferron et al. reported that knockout of the insulin receptor on osteoblasts led to low bone mass in mice (1). By secreting osteocalcin (OC) osteoblasts participate in bone formation as well as energy metabolism. Osteocalcin in carboxylated form (cOC) interacts with hydroxyapatite crystals of the bone matrix $(2,3)$. During bone resorption, undercarboxylated osteocalcin (ucOC) is released and participates in glucose and lipid metabolism $(4,5)$. Studies in mice showed that knockout of the osteocalcin gene (Ocn-/-) led to obesity, increased serum triglycerides as well as suppression of insulin secretion and glucose intolerance (6). Human studies also showed that obese subjects with pre-diabetes had decreased ucOC blood levels, which

Published by Bioscientifica Ltd. 
was inversely associated with HOMA-IR and serum insulin $(7,8)$.

Recent studies focused on the circulatory component of bone remodelling $(9,10,11,12)$, which appears to be tightly controlled by osteoblasts, osteocytes, immune cells, and the endocrine system. Namely, as regards bone formation, studies report that mesenchymal stem cells (MSC) from which osteoblasts are differentiated could be obtained not only from bone marrow but also from peripheral blood (9). It has been documented that osteoblast-lineage cells were present in the circulation of adult subjects and were associated with biochemical markers of bone formation (10). Circulating monocytes in turn could be considered cell cycle-arrested quiescent osteoclast precursors, which start their differentiation in haematopoietic tissues, afterwards circulate in the bloodstream, and then migrate to bones for final osteoclastogenesis $(11,12,13,14,15)$. Taking into consideration the circulatory component of the process of bone formation and resorption, gene expression patterns in whole blood could partially reflect bone turnover processes in healthy as well as disease states.

In our study we concentrated on the effect of insulin resistance and disturbed oral glucose insulin sensitivity on the expression of mRNA and miRNA affecting bone homeostasis in whole blood from obese individuals as well as the influence of oral glucose load on serum osteocalcin concentration in obesity. We hypothesise that due to insulin resistance, the expression of mRNA and miRNA affecting bone turnover could be disturbed and consequently, blood osteocalcin concentration after OGTT could differ in comparison to subjects without insulin resistance.

\section{Subjects and methods}

\section{Subjects}

The cross-sectional study was performed in accordance with The Code of Ethics of the World Medical Association (Declaration of Helsinki) and was approved by the Bioethics Committee of the Jagiellonian University in Cracow, Poland (opinion number KBET/82/B/2009). Study participants were recruited from patients at the Outpatient Clinic of Obesity and Lipid Disorder Treatment at the Department of Biochemistry, Jagiellonian University Medical College in Cracow, Poland from September 2009 till July 2013. Prior to participation in the study written consent was obtained from each subject after full explanation of the purpose and nature of procedures used. Overweight and obese $\left(25 \leq \mathrm{BMI}<40 \mathrm{~kg} / \mathrm{m}^{2}\right)$ women and men, aged 25-65 years were included into the study. Subjects suffering from chronic diseases (cardiovascular diseases, cancer, and chronic inflammation), diabetes mellitus and other metabolic disorders, impaired kidney or liver function were excluded from the study. Of the 195 participants recruited for the study, 50 declined to participate and 63 subjects did not meet the inclusion criteria. Thus, the study included 82 participants. Subjects included in the study were divided into two groups depending on oral glucose insulin sensitivity index (OGIS) (16): subjects without insulin resistance (control group with OGIS values $>392 \mathrm{~mL}^{*} \mathrm{~min}^{-1 *} \mathrm{~m}^{-2}$, $n=41$ ) and the group with insulin resistance (OGIS values $\left.\leq 392 \mathrm{~mL}^{*} \mathrm{~min}^{-1 *} \mathrm{~m}^{-2}, n=41\right)$. All subjects underwent anthropometrical measurements: body mass index (BMI), waist-to-hip ratio (WHR) and body composition estimated with the bioelectrical impedance method using the Segmental Body Composition Analyser TANITA (Tanita, Tokyo, Japan).

\section{Oral glucose tolerance test}

Participants underwent a 2-h oral glucose tolerance test (OGTT) conducted according to the World Health Organization (WHO) and International Diabetes Federation (IDF) guidelines. Tests were performed in the morning hours (8:00-10:00 h) after 10-h overnight fasting. Blood samples were collected at baseline (fasting), 60, and $120 \mathrm{~min}$ after the ingestion of $75 \mathrm{~g}$ glucose dissolved in $250 \mathrm{~mL}$ of water. All patients were asked not to drink alcohol or drinks containing caffeine for 3 days before the test.

\section{Biochemical tests}

Serum from blood samples collected before the OGTT was used for biochemical tests: total cholesterol, HDL cholesterol and inflammatory markers (CRP, IL-6). Total and HDL cholesterol were measured by enzymatic colorimetric methods using the MaxMat Analyzer (Maxmat SA, Montpellier, France). LDL cholesterol was calculated using the Friedewald formula. IL-6 was measured using ELISA (R\&D Systems Europe, Ltd). CRP was measured by immunoturbidimetric method (APTEC Diagnostics nv, Sint-Niklaas, Belgium). In blood samples collected during the OGTT glucose, insulin, non-esterified free fatty acids (NEFAs), triglycerides (TGs), cOC and ucOC were measured. Serum glucose and triglycerides were measured by enzymatic colorimetric methods on 
the MaxMat Analyzer. Serum insulin was measured by immunoradiometric method (DIAsource, ImmunoAssays, Louvain-la-Neuve, Belgium) using the gamma counter (LKB Instruments, Mount Waverley, Australia). Basal insulin resistance was estimated using homeostasis model of assessment (HOMA-IR) (17). To assess post oral glucose load insulin sensitivity, an oral glucose insulin sensitivity index (OGIS) proposed by Mari et al. (16) was used. OGIS was computed using a calculator for Excel spread sheet available on the website http://webmet.pd.cnr.it/ogis. Serum $\mathrm{COC}$ and ucOC were determined by ELISA (Takara, Shiba, Japan). Total osteocalcin level was calculated as the sum of COC and ucOC. NEFAs concentration was measured immediately in non-frozen plasma by enzymatic quantitative colorimetric method (Roche Diagnostics $\mathrm{GmbH})$.

\section{Sample collection, RNA and miRNA isolation}

For analysis of gene expression and miRNAs, venous blood before OGTT (fasting sample) was collected into PAXgene Blood RNA Tubes (Becton Dickinson), following a 2-h room temperature incubation, samples were frozen at $-80^{\circ} \mathrm{C}$. Total RNA, miRNA enriched, was isolated using the PAXgene 96 Blood RNA Kit and GeneMATRIX Universal RNA/miRNA Purification Kit. RNA and miRNA quality were assessed in an Agilent Bioanalyzer 2100 using the RNA 6000 Nano kit (Agilent Technologies) and quantified by spectrophotometry using a NanoDrop ND-1000 UV-VIS spectrophotometer (Thermo Fisher Scientific). Samples with RIN $>7.0$ were selected for further analysis.

\section{Quantification of mRNA transcripts}

Gene expression profiles were determined using Single Color 39494 human oligonucleotide arrays and reagents from Agilent Technologies. Each separate RNA sample was hybridised to a single array. Labelling of $100 \mathrm{ng}$ of total RNA was done using the Quick Amp labelling kit according to the manufacturer's protocol. RNA concentration and dye incorporation were measured using Nanodrop ND-1000. Signal intensity of the labelled cRNA was measured by hybridisation to a human gene microarray. Detection of the fluorescence signal was performed by the Agilent SureScan Microarray Scanner using extraction software (Agilent Technologies). For microarray hybridisation, nine samples were used (four samples from the control group, five samples from obese subjects with insulin resistance). Microarray data analysis was performed using Gene Spring version 13 (Agilent Technologies). To identify differentially expressed genes each data set of fluorescence signals was normalised using median shift algorithm (shifted to 75 percentile) with background correction to the median of all samples. Normalised signal was used for fold of change (FC) calculation. FC was calculated in relation to obese with insulin resistance versus obese without insulin resistance and used as expression level.

\section{Quantification of microRNA (miRNA, miR) transcripts by TLDA}

For relative quantification of miRNA by Real-time PCR, TaqMan Low-Density Array (TLDA) was used (Thermo Scientific). Reverse transcription was performed with TaqMan MicroRNA Reverse Transcription Kit and MegaPlex Human Pool A RT primers. Preamplification was enabled by Megaplex ${ }^{\mathrm{TM}}$ PreAmp Kit and Primers. TaqMan ${ }^{\circledR}$ Array Human MicroRNA A+B Cards Set v3.0 were employed for the accurate quantitation of 754 human microRNA by real-time PCR. The arrays were run in 7900 HT Fast Real-Time PCR system (Thermo Scientific). Relative miRNA levels were calculated using DataAssist v 3.01 tool and expressed as $\mathrm{RQ}=2^{\wedge}(-\Delta \mathrm{Ct})$ obese with insulin resistance $/$ $2^{\wedge}(-\Delta \mathrm{Ct}$ )obese without insulin resistance. RNU44, RNU48 and U6 snRNA were used as endogenous controls.

\section{Statistical analysis}

Analyses of nominal data were performed using chisquare $\left(\chi^{2}\right)$ test. The Shapiro-Wilk test was used to analyse data for a Gaussian distribution. For verification of homogeneity of variance Levene's test was used. Continuous variables were $\log$ transformed if required. Normally distributed data are presented as mean \pm S.E.M. otherwise as median and lower-upper quartile range (25$75 \%)$. Differences between obese with insulin resistance versus obese without insulin resistance were calculated by unpaired $t$-test or Mann Whitney $U$ test (for nonnormally distributed data). Potential differences in serum glucose, insulin, NEFAs, triglycerides and osteocalcins during OGTT were calculated with repeated-measures ANOVA. Values at single time points were compared by Tukey post hoc test. $P$ values less than 0.05 were considered significant. Statistical analyses were performed with the Statistical software (StatSoft Polska, Krakow, Poland). The list of significantly regulated transcripts in whole blood of obese with impaired insulin sensitivity versus obese without insulin resistance with $P<0.05$ was generated using statistical filtering (Moderated $T$-test with Benjamini-Hochberg multiple test correction). 
Analysis of regulated pathways was performed using PathVisio 3 software (https://www.pathvisio.org).

$P$ value for differences in miRNA expression in whole blood between obese with insulin resistance versus obese without insulin resistance was calculated using $t$-test as comparison of the normalised $2^{(-\Delta \mathrm{Ct})}$ values of the two groups. The comparison of biochemical measurements as well as regulated transcripts and miRNA expression were not adjusted for confounders because obese with and without insulin resistance were not statistically different regarding possible confounders such as sex, age, BMI and adipose tissue mass.

\section{Results}

\section{Characteristics of the subjects}

The basal characteristics of participants is summarised in Table 1. Both groups of subjects (subjects with insulin resistance $n=41$ vs individuals without insulin resistance $n=41$ ) did not differ significantly regarding age, sex, BMI, waist circumference, WHR and adipose tissue mass. However, in comparison to control group (subjects without insulin resistance), the insulin resistance subjects had higher fasting serum glucose $(5.31 \pm 0.08$ vs $5.06 \pm 0.08 \mathrm{mmol} / \mathrm{L}(P=0.018))$ and higher HOMA-IR value $(4.04(2.41-5.46)$ vs $2.60(2.15-3.67)(P=0.027))$. During OGTT 1-h serum glucose was higher in the group of subjects with insulin resistance than controls $(9.08 \pm 0.38$ vs $7.90 \pm 0.39 \mathrm{mmol} / \mathrm{L}, P=0.001)$ (Fig. $1 \mathrm{~A})$. In these subjects neither fasting nor 2-h mean serum glucose was above the upper reference limit: 5.55 and $7.8 \mathrm{mmol} / \mathrm{L}$, respectively. However, 12 subjects had elevated fasting serum glucose $(5.6-6.4 \mathrm{mmol} / \mathrm{L})$. Impaired glucose tolerance was observed in 9 individuals with 2-h serum glucose between 7.8 and $10.5 \mathrm{mmol} / \mathrm{L}$. Subjects with insulin resistance had higher serum insulin in comparison to control group $(P=0.016)$. Serum insulin increased $1 \mathrm{~h}$ after glucose load by about $500 \%$ and then gradually fell

Table 1 Basal characteristics of subjects included in the study.

\begin{tabular}{l}
\hline \\
\hline Age (years) \\
Sex, female (\%) \\
BMI (kg/m²) \\
WC (cm) \\
$\quad$ Females \\
$\quad$ Males \\
WHR \\
$\quad$ Females \\
$\quad$ Males \\
Adipose tissue mass (\%) \\
Systolic BP (mm Hg) \\
Diastolic BP (mm Hg) \\
Total cholesterol (mmol/L) \\
HDL cholesterol (mmol/L) \\
LDL cholesterol (mmol/L) \\
NEFA (mmol/L) \\
Triglycerides (mmol/L) \\
Glucose (mmol/L) \\
Insulin (ulU/mL) \\
HOMA-IR \\
IL-6 (pg/mL) \\
CRP (mg/L) \\
Vitamin D (ng/mL) \\
ucOC (ng/mL) \\
cOC (ng/mL) \\
Total OC (ng/mL) \\
\hline
\end{tabular}

\begin{tabular}{c} 
Obese with insulin resistance $(n=41)$ \\
\hline $48.32 \pm 1.63^{\dagger}$ \\
68 \\
$33.10(31.12-35.54)^{\ddagger}$ \\
$99(92.8-106.5)$ \\
$115(109.5-121)$ \\
$0.84(0.82-0.91)$ \\
$1.02(0.99-1.04)$ \\
$38.60(33.35-41.55)$ \\
$130(120-140)$ \\
$84(80-90)$ \\
$5.49 \pm 0.16$ \\
$1.27 \pm 0.04$ \\
$3.50 \pm 0.14$ \\
$0.72 \pm 0.03$ \\
$1.69 \pm 0.12$ \\
$5.31 \pm 0.08$ \\
$17.38 \pm 1.40$ \\
$4.04(2.41-5.46)$ \\
$1.62 \pm 0.19$ \\
$2.18(1.00-3.81)$ \\
$13.24(10.48-18.64)$ \\
$3.57 \pm 0.37$ \\
$5.47 \pm 0.29$ \\
$9.03 \pm 0.52$ \\
\end{tabular}

Obese without insulin resistance $(n=41)$
$45.68 \pm 1.88$
80
$32.08(28.98-34.82)$
$96(90-101.5)$
$114(102-116)$
$0.82(0.80-0.85)$
$0.98(0.95-0.99)$
$38.80(33.85-41.70)$
$122(116-130)$
$81(76-90)$
$5.50 \pm 0.17$
$1.29 \pm 0.03$
$3.59 \pm 0.16$
$0.78 \pm 0.04$
$1.39 \pm 0.11$
$5.06 \pm 0.08$
$14.50 \pm 1.20$
$2.60(2.15-3.67)$
$1.33 \pm 0.12$
$1.53(0.63-4.07)$
$16.71(9.36-24.57)$
$3.04 \pm 0.28$
$6.21 \pm 0.42$
$9.25 \pm 0.51$

\begin{tabular}{l}
\hline \multicolumn{1}{c}{$\boldsymbol{P}^{*}$} \\
\hline 0.292 \\
$>0.05$ \\
0.124 \\
\\
0.281 \\
0.237 \\
\\
0.113 \\
0.024 \\
0.954 \\
0.298 \\
0.438 \\
0.945 \\
0.646 \\
0.670 \\
0.432 \\
0.049 \\
0.018 \\
0.088 \\
0.027 \\
0.224 \\
0.919 \\
0.207 \\
0.446 \\
0.137 \\
0.291 \\
\hline
\end{tabular}

*Significant difference between obese with insulin resistance and obese without insulin resistance, (unpaired $t$-test or Mann-Whitney $U$ test for non-normally distributed variables; nominal data were analysed by $\chi^{2}$ test), $P<0.05$. ${ }^{\dagger}$ Mean \pm S.E.M.; all such values. ${ }^{\ddagger}$ Median (25-75\%); all such values. BMI, body mass index; BP, blood pressure; COC, carboxylated osteocalcin; HDL, high-density lipoprotein; HOMA-IR, homeostatic model assessment; CRP, C-reactive protein; IL-6, interleukin 6; LDL, Iow-density lipoprotein; NEFA, non-esterified fatty acids; OC, osteocalcin; ucOC, undercarboxylated osteocalcin; WC, waist circumference; WHR, waist-to-hip ratio. 

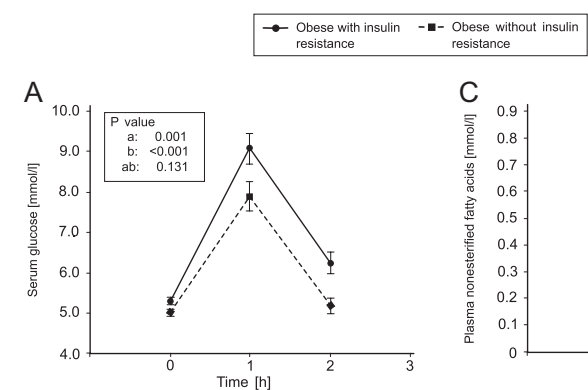

B
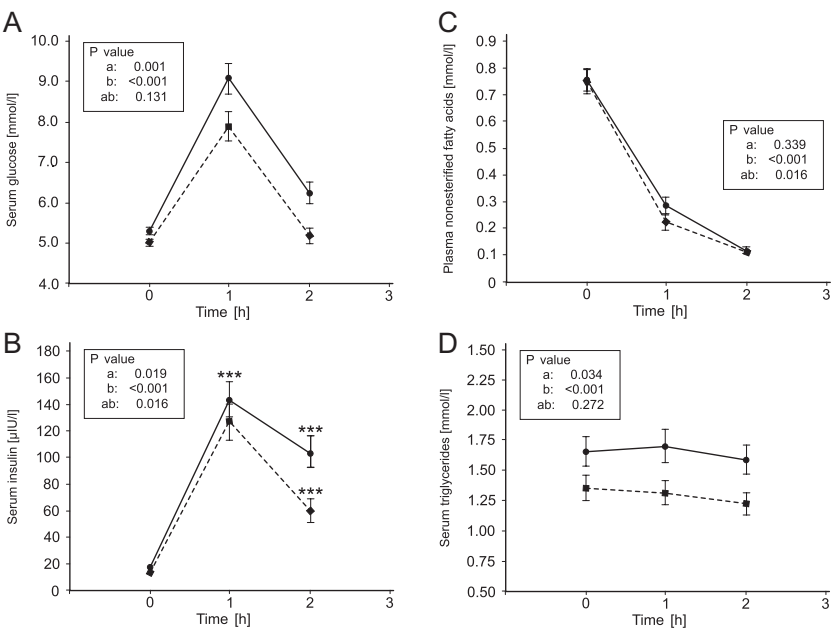

\section{Figure 1}

Blood levels of glucose (A), insulin (B), non-esterified fatty acids (NEFAs) (C) and triglycerides (D) during oral glucose tolerance test. Data are presented as means \pm S.E.M. Statistical analyses were performed with repeated-measures ANOVA and denote differences between obese with insulin resistance and obese without insulin resistance participants (a), differences over time (b) and differences owing to interaction between group and time (ab). Values at single time points were compared by Tukey post hoc tests. Significant differences are indicated as follows: $* * * P<0.001$.

after $2 \mathrm{~h}$ by about $20 \%$ in the group with insulin resistance and approximately by $50 \%$ in the control group (Fig. 1B). There were no statistically significant differences in serum LDL, HDL and total cholesterol between studied groups except for serum triglycerides $(1.69 \pm 0.12$ vs $1.39 \pm 0.11$, $P=0.049$ ).

As regards plasma NEFAs in response to glucose load, we found no statistically significant differences between the studied groups $(P=0.309)$. In both studied groups plasma NEFAs gradually decreased after 1 and $2 \mathrm{~h}$ of the test in comparison to baseline values $(P<0.001)$ (Fig. 1C). However, we found that during OGTT serum triglycerides concentration was higher in the group of subjects with insulin resistance than controls $(P=0.034)$ (Fig. 1D). We did not observe any statistically significant differences between the studied groups in serum inflammatory markers: IL-6 (1.62 \pm 0.19 vs $1.33 \pm 0.19 \mathrm{pg} / \mathrm{mL}, P=0.224)$, CRP $(2.18$ (1.00-3.81) vs $1.53(0.63-4.07) \mathrm{mg} / \mathrm{L}, P=0.919)$, which probably was connected with similar BMI, WHR and adipose tissue mass in both groups of patients (Table 1).

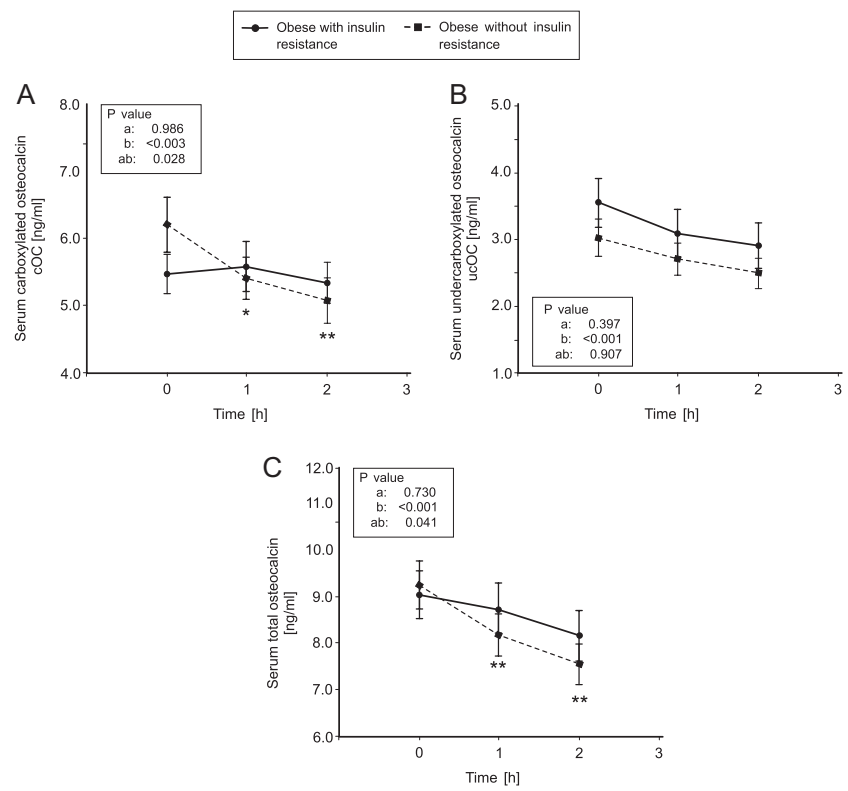

\section{Figure 2}

Blood concentrations of carboxylated osteocalcin (cOC) (A), undercarboxylated osteocalcin (ucOC) (B) and total osteocalcin (C) during oral glucose tolerance test. Data are presented as means \pm S.E.M. Statistical analyses were performed with repeated-measures ANOVA and denote differences between obese with insulin resistance and obese without insulin resistance participants (a), differences over time (b) and differences owing to interaction between group and time (ab). Values at single time points were compared by Tukey post hoc tests. Significant differences are indicated as follows: $\star P<0.05, * * P<0.01$.

\section{Effects of insulin resistance in obesity on serum osteocalcin during OGTT}

Although baseline serum osteocalcin (cOC) was similar in both studied groups $(5.47 \pm 0.19$ vs $6.21 \pm 0.19 \mathrm{ng} / \mathrm{mL}$, $P=0.139$ ) (Table 1$)$, the response to oral glucose load was different in respect to serum cOC $(P=0.028)$ (Fig. $2 \mathrm{~A})$. Surprisingly, in subjects without insulin resistance serum cOC gradually decreased after $1 \mathrm{~h} \quad(5.41 \pm 0.32$ vs $\quad 6.21 \pm 0.19 \mathrm{ng} / \mathrm{mL}, \quad P<0.05)$ and $2 \mathrm{~h} \quad(5.06 \pm 0.32$ vs $6.21 \pm 0.19 \mathrm{ng} / \mathrm{mL}, P<0.01)$ of OGTT compared to baseline values. This effect was suppressed in subjects with insulin resistance. Similar to serum cOC, total osteocalcin concentration in control group but not in subjects with insulin resistance $(P=0.041)$ was gradually reduced at $1 \mathrm{~h}(8.17 \pm 0.44$ vs $9.25 \pm 0.44 \mathrm{ng} / \mathrm{mL}, P<0.01)$ and $2 \mathrm{~h}(7.53 \pm 0.43$ vs $9.25 \pm 0.44 \mathrm{ng} / \mathrm{mL}, P<0.01)$ after glucose load (Fig. 2C). However, no statistically significant differences were observed in serum ucOC during OGTT 
between the studied groups. In comparison to baseline values, both groups of subjects presented gradually reduced serum ucOC levels during $1 \mathrm{~h}$ and $2 \mathrm{~h}$ of OGTT $(P<0.001)$ (Fig. 2B).

\section{Effects of insulin resistance in obesity on the expression of mRNA and miRNA participating in the regulation of osteoblasts and osteoclasts differentiation}

Results of gene expression analysis in whole blood of obese subjects with insulin resistance are presented in Table 2. Analysis of regulated pathways performed by PathVisio 3 software revealed that two groups of studied subjects differ regarding following pathways: osteoclast signalling, osteoblast signalling, Wnt Signalling pathway, vitamin D receptor pathway, TGFb signalling pathway, insulin signalling, cytokines and inflammatory response.

Obese subjects with insulin resistance in comparison to control group presented inhibition of insulin signalling pathway genes expression in whole blood. In comparison to subjects without insulin resistance, they had downregulation of receptors for IGF1 (IGF1R), P13-Kinase Isoforms (PIK3CD, PIK3CG, PIK3C3, PIK3R1, PIK3R2), genes participating in PDK/AKT signalling pathway (PDPK1, AKT2, GSK3A, GSK3B) as well as transcription factor FOXO1. As regards cytokines and inflammatory response, we found that expression of genes coding for cytokines/antigens taking part in inflammatory response was upregulated: IL6, IL12B, IL12A. The mRNA levels of genes involved in the regulation of chemotaxis were increased in subjects with insulin resistance versus individuals without insulin resistance: CCL2, CXCL8, CXCL10, CXCL9, CXCL11. Expression of other proinflammatory cytokines was also upregulated: IFNA10, IFNA21, IFNA14, IFNB1, IFNA16, IFNA6, IFNA7, IFNB1. Analysis of gene expression in subjects with insulin resistance also revealed upregulation of genes coding for CRP, CSF1 (MCP1), receptor for IL-1 (IL1R1) as well as co-stimulatory molecules: CD40, CD80.

The mRNA levels of genes responsible for osteoblasts signalling differentiation and maturation were also differentially regulated in obese with insulin resistance in comparison to subjects without insulin resistance. The expression in whole blood of genes being early markers of osteoblasts differentiation was statistically significantly regulated in obese subjects. We found downregulation of alkaline phosphatase $(A L P L)$ and upregulation of osteopontin (SPP1), bone sialoprotein (IBSP), type 1 alpha collagen (COL1A1) in obese subjects with insulin resistance. The expression of osteocalcin gene (BGLAP) was unchanged (data not shown). Among factors which modulate osteoblast differentiation, we observed downregulation of TGFB1, receptor for vitamin $\mathrm{D}(V D R)$, but mRNA for bone morphogenic proteins (BMP2, BMP4, $B M P 7)$ and the receptor for PTH (PTH1R) were upregulated in insulin resistance subjects. Analysis of gene expression also revealed upregulation of BMP antagonist GREM2 and TGFB3 as well as inhibition of the receptor for TGFB (TGFBR2) in obese subjects with insulin resistance. However, the expression of Wnt signalling antagonists: DKK1, DKK2, DKK4, sclerostin (SOST), SFRP1, DUSP2, $A C V R 2 A$ and WIF1 was upregulated. We also found upregulation of Wnt signalling agonists WNT3A, WNT5A and FZD and their receptor LRP5. However, CTNNB1 and its nuclear partners $L E F-1 / T C F 4$ transcription factor family were downregulated in obese subjects with insulin resistance. Expression of key transcription factors in osteoblast differentiation (RUNX2, osterix SP7, MSX2, DLX5, TWIST1 and TWIST2) was upregulated. However, expression of RUNX2 degradation protein gene (SMURF2) was upregulated. Expression of another osteoblast differentiation factor ATF4 was inhibited in whole blood of subjects with insulin resistance as compared to subjects without insulin resistance.

The mRNA levels of genes participating in osteoclasts signalling differentiation were statistically significantly upregulated in obese subjects with insulin resistance. As regards RANKL/RANK/OPG pathway, we found upregulated expression of TNFSF11A (RANK), TNFRSF11B (Osteoprotegerin) and TNSF11 (RANKL). However, expression ratio of TNSF11/TNFRSF11B was 1. Although gene expression of CSF1 (MCP1) was increased, we observed inhibition of SPI1 (PU.1). Moreover, gene expression of NFATC1 as well as regulated osteocytes' genes, such as tartrate-resistant acid phosphatase (ACP5 (TRAP), ITGA5), was downregulated.

Expression of miRNAs participating in regulation of osteoblasts and osteoclasts differentiation is shown in Table 3. We found significant downregulation of miR-324-3p in obese subjects with insulin resistance compared to volunteers without insulin resistance. It was demonstrated that miR-324-3p expression is inhibited in subjects with type 2 diabetes and is associated with disturbances in bone microstructure $(18,19)$. In blood of obese subjects with insulin resistance, miR-181a expression was also downregulated, which could negatively affect osteoblastic differentiation due to upregulation of BMP antagonist expression (GREM2). Contrary to control group, insulin-resistant subjects had decreased expression 


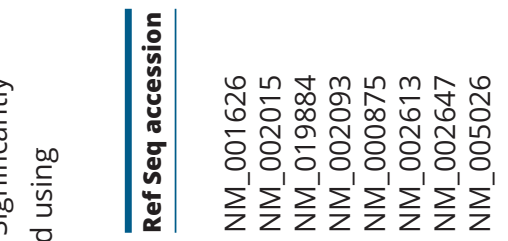

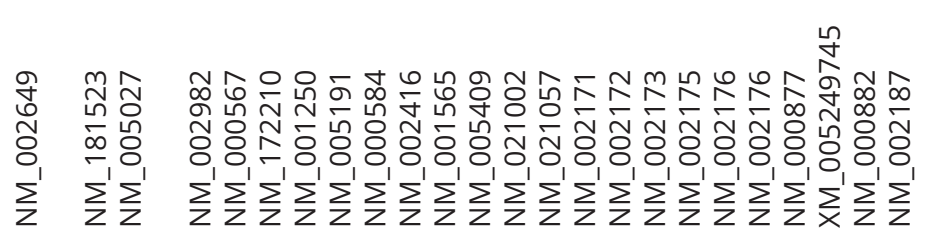

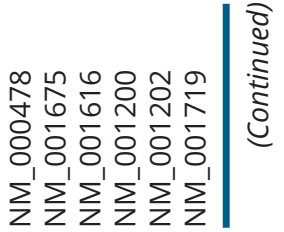

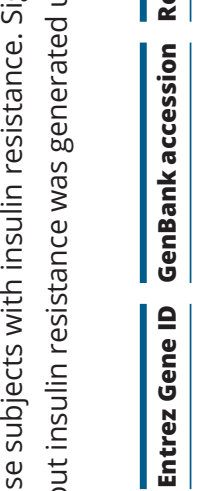

ஸै

ปับ Nㅗㅇ

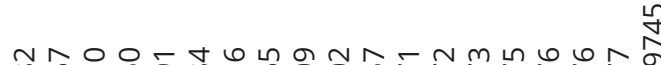

ᄂ

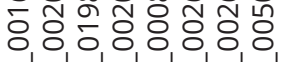

กิ

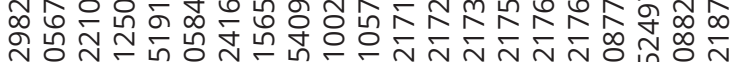

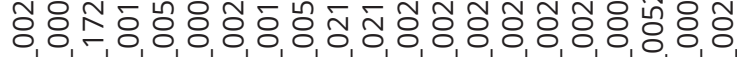

$\sum \sum \sum \sum \sum \sum \sum$

$\sum \sum \sum$

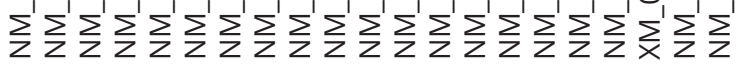

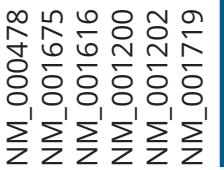

ᄋ

กิ กิ กั

チธํํำ

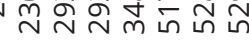

mे

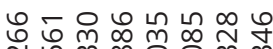

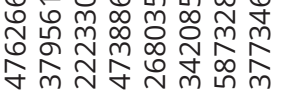

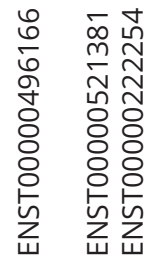

$\bar{\delta} \frac{\bar{\sigma}}{\bar{\sigma}}$

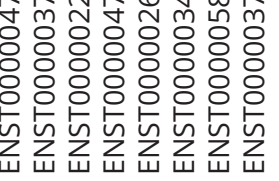

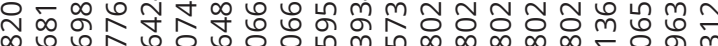

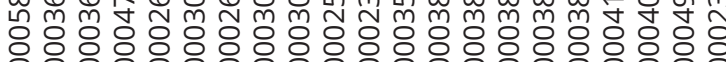

80000

느느ํ는는

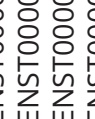

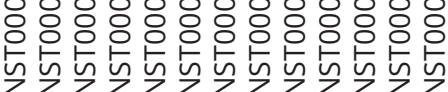

ш

$\left|\begin{array}{l}\frac{1}{0} \\ 0 \\ 0\end{array}\right|$

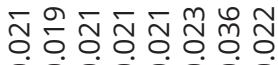

ปั

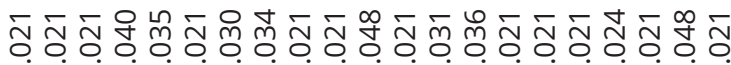

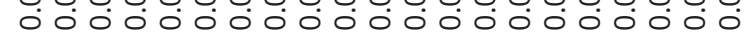

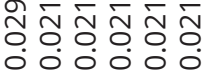

u

뉴용ำ

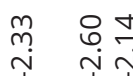

m

$\hat{N} \stackrel{\infty}{m} \stackrel{\infty}{\sim} \stackrel{m}{\sim} \stackrel{\sim}{\sim} \stackrel{\sim}{N}$

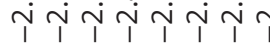

î

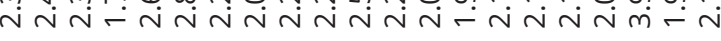

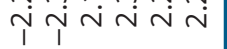

$\stackrel{0}{\stackrel{0}{a}}$

$\subseteq \frac{0}{0}$

गे

?

응

응 을

$\frac{0}{3} \stackrel{\subseteq}{\frac{1}{3}}$

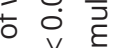

$\tilde{a}$, o

䆛

8 这

荡

읒 है

बें है

츰

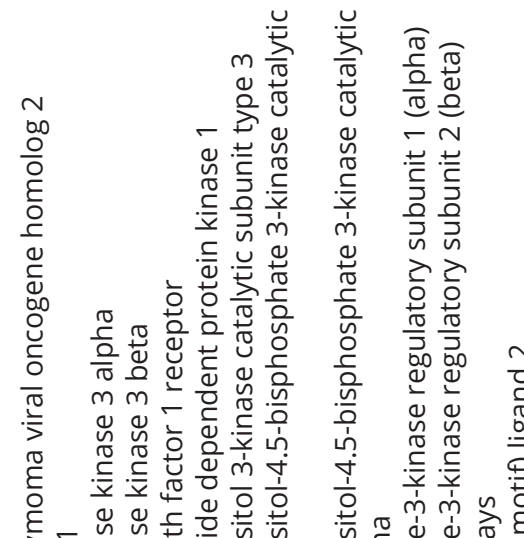

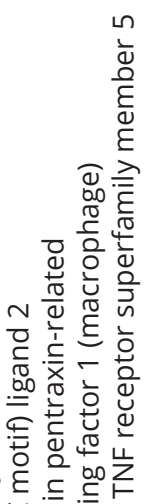

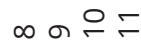

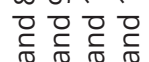

兽

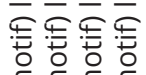

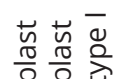

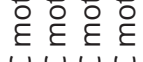

응 응

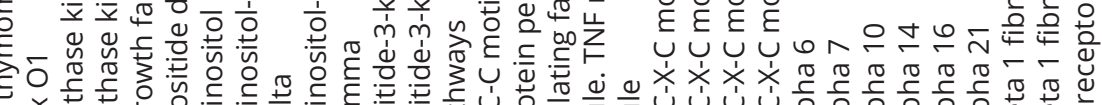

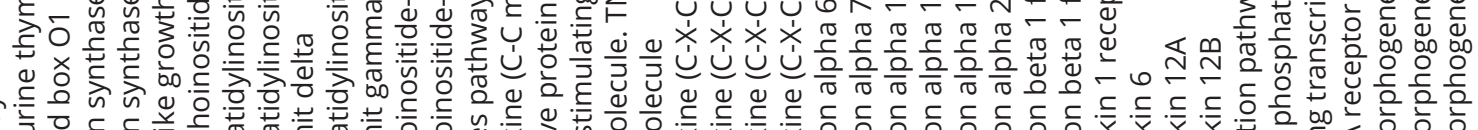

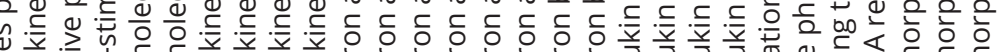

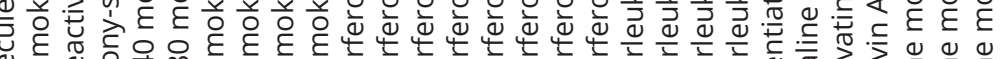

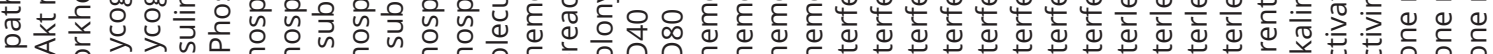

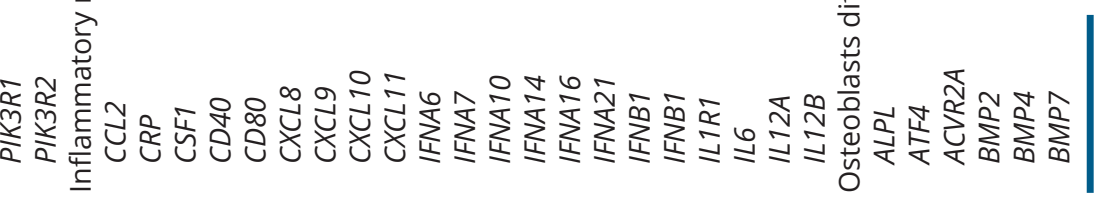




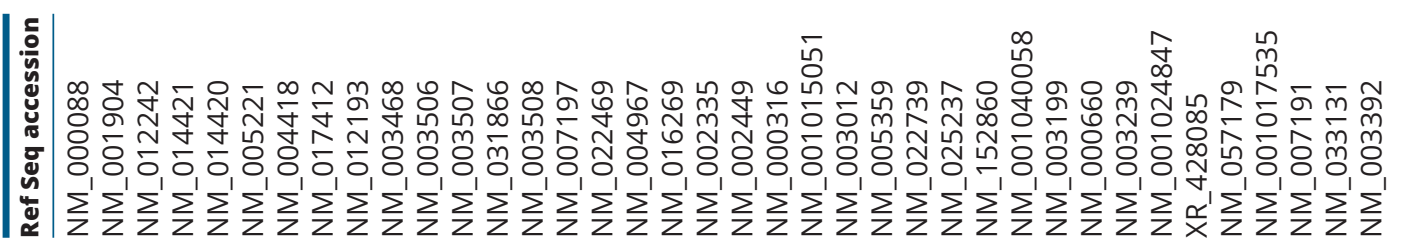

ᄃ유에

늄

88 단

$\sum \sum \sum$

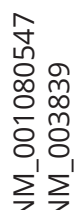

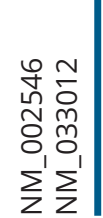

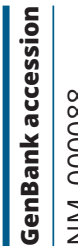

约

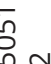

品

今े

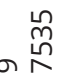

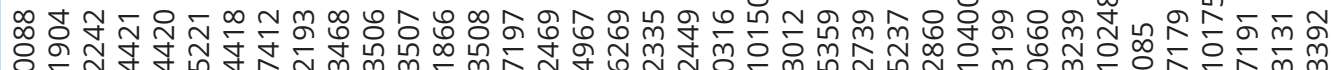

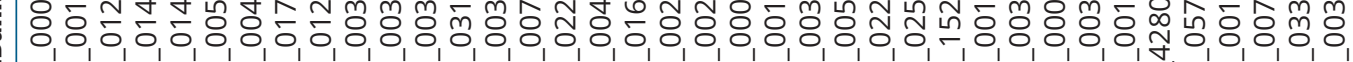

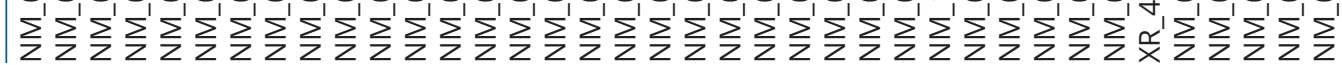

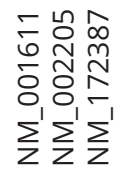

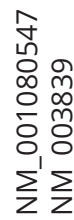

เั้

ผิ

ठํำ

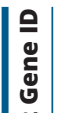

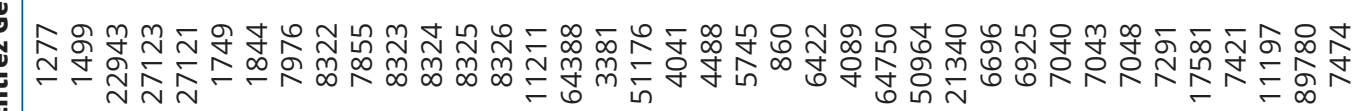

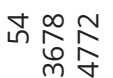

\begin{tabular}{l}
$\infty$ \\
$\infty$ \\
$\substack{\widehat{N} \\
\hdashline}$
\end{tabular}

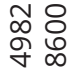

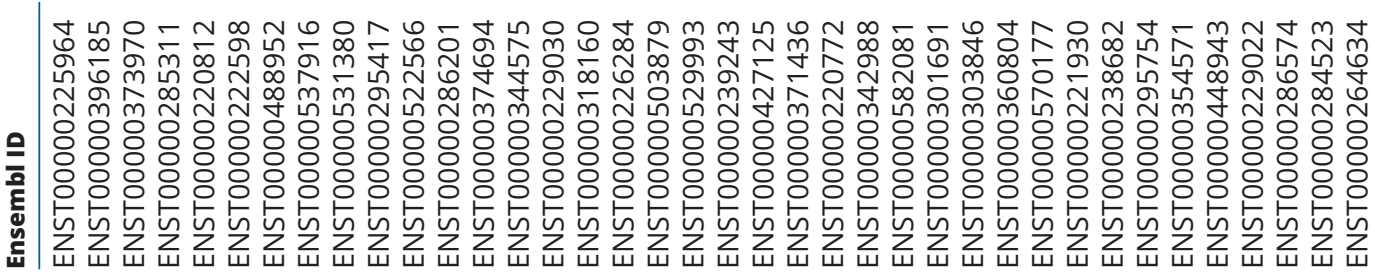

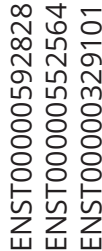

요요

$\stackrel{5}{\wedge}$

สิ

○

은

$\sum \sum$

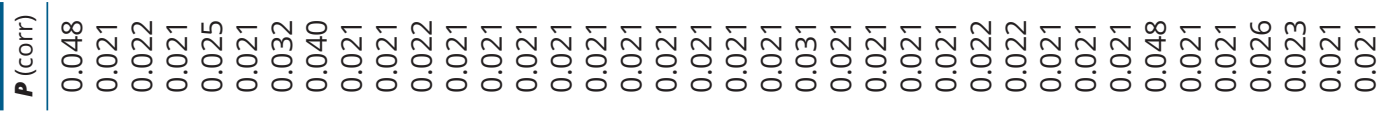

웡 $\bar{d}$

บ

政

ลิ เุ

$\overleftarrow{\sim}$

$\varnothing$

量空

$\sum_{u}^{n}$

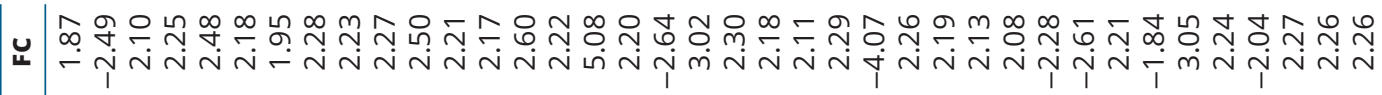

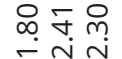

ธิธ

ธิธิ์

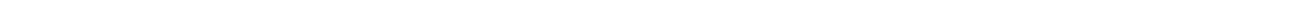

i

옥

mo i

i่

$\stackrel{0}{0}$
$\stackrel{2}{\infty}$
$\infty$
$\infty$

d.

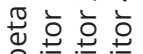

을 을

.

잋 तो

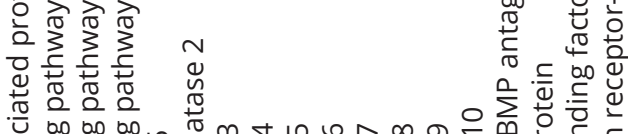

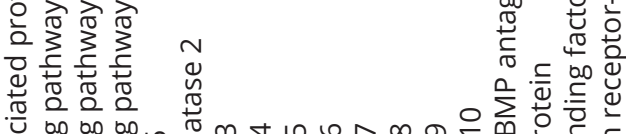

-

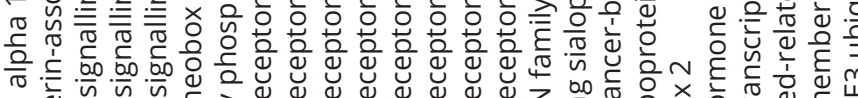

ه

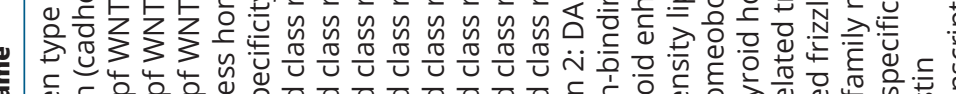

焉

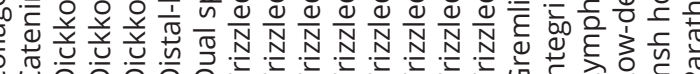

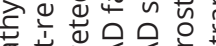

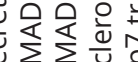

哭氐

हैं

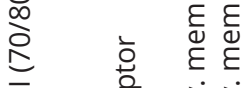

=

产

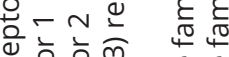

育袬

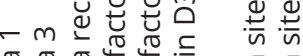

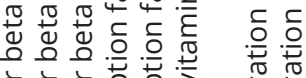

$\pi$

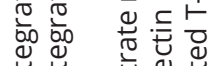

市

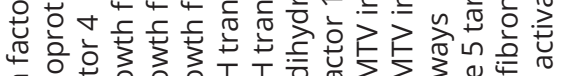

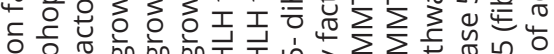

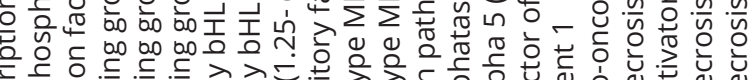

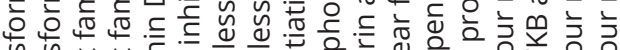

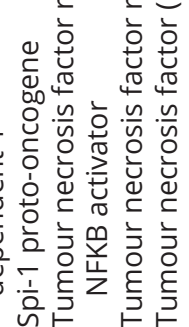

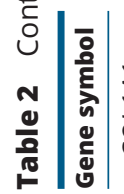

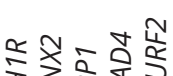
政 के

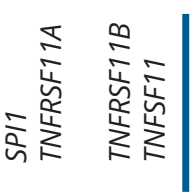


Table 3 Relative quantification (RQ) of miRNA expression profile in obese subjects with insulin resistance versus obese without insulin resistance individuals.

\begin{tabular}{|c|c|c|}
\hline miRNA & $\mathbf{R Q}$ & $P$ value \\
\hline hsa-miR-29b & 0.474 & 0.046 \\
\hline hsa-miR-181a & 0.297 & 0.009 \\
\hline hsa-miR-210 & 0.458 & 0.035 \\
\hline hsa-miR-324-3p & 0.467 & 0.012 \\
\hline
\end{tabular}

$P$ value for differences in miRNA expression in whole blood between obese with insulin resistance and obese without insulin resistance subjects was calculated using Student's $t$-test as comparison of the normalised $2^{(-\Delta C t)}$ values of the two groups.

of miR-210 in whole blood, which can indicate that osteoblast proliferation and differentiation were inhibited. Moreover, we found decreased miR-29b expression, which could contribute to osteogenesis disturbances due to increased expression of osteoblast differentiation inhibitors (TGFB3, ACVR2A, DUSP2) (20).

\section{Discussion}

The study is the first one focusing on both mRNA and microRNA in whole blood, associated with bone remodelling in obese subjects with and without insulin resistance. Analysis of gene expression was performed in whole blood. It is well known that RNA information from whole blood resides in circulating blood leukocytes. However, when analysis of gene expression connected with bone turnover is performed in whole blood, circulatory component of bone remodelling should be taken into consideration. Namely, osteoblast-lineage cells circulate in peripheral blood in physiologically significant numbers which correlate with markers of bone formation (10). Osteoclast precursors are also detectable in the peripheral circulation (11).

In comparison to the control group (subjects without insulin resistance), the study group had a higher degree of insulin resistance determined by lower OGIS and higher HOMA-IR values. Studied subjects with insulin resistance had no diabetes but 12 of 41 subjects (29\%) had impaired fasting glucose $(5.6-6.4 \mathrm{mmol} / \mathrm{L})$ and nine subjects $(22 \%)$ had impaired glucose tolerance (2-h serum glucose $7.8-10.5 \mathrm{mmol} / \mathrm{L})$, together 17 subjects (41\%) had pre-diabetes. Obese insulin-resistant subjects had higher basal and post-glucose load serum insulin, glucose and triglycerides in comparison to the control group. Analysis of gene expression in whole blood from obese subjects with insulin resistance confirmed insulin signalling pathway defects. We observed downregulation of $I G F 1 R$,
P13-kinase isoforms, genes participating in PDK/AKT signalling pathway. It should be stressed that both groups did not differ regarding BMI, adipose tissue mass as well as serum inflammatory markers.

Some studies reported that osteoblasts from obese insulin resistant subjects were less active and produced less collagen as well as had lower bone formation index defined as quotient of procollagen type 1 amino-terminal propeptide to bone alkaline phosphatase (PINP/BAP) (21). In our studies we also reported that disturbances in insulin signalling could affect osteoblastogenesis by inhibiting expression of genes connected with Wnt, BMP signalling pathway and upregulation of genes participating in inflammatory response. Indeed, we found downregulation of $\beta$-catenin (CTNNBI), which via mediating WNT signal transduction, plays a key role in the commitment of early progenitors to osteoblast precursors. Although we observed upregulation of WNT signalling pathway agonists (WNT3A, WNT5A and $F Z D)$ as well as their receptor LRP5, the expression of this pathway antagonists was also upregulated (SFRP, WIF-1, SOST, DKK1, DKK2). WNT ligands can interact with antagonists, including (SFRP) family and WNT inhibitory factor 1 (WIF1), preventing activation of the pathway. In turn LRP5/6 co-receptor activity is inhibited by SOST (sclerosteosis gene product) (22) and Dickkopf (DKK) families $(23,24)$, all of which bind LRP5/6, that in consequence leads to the downregulation of $\beta$ catenin gene expression. Although we observed the upregulation of gene expression for BMPs promoting bone formation, such as BMP2, BMP4, BMP6 (25), the gene expression of SMAD4 in BMP signalling was downregulated in subjects with insulin resistance. It has been reported previously that conditional deletion of SMAD4 in osteoblasts, leads to lower bone mineral density, decreased bone volume, decreased bone formation rate, and a reduced number of osteoblasts (26).

Although gene expression of key transcription factor in osteoblast differentiation - RUNX2 was upregulated in obese subjects with insulin resistance, its protein concentration could be decreased by upregulation of SMURF2 (27) as well as TWIST1 and TWIST2 (28). Bialek et al. showed that RUNX2-induced osteoblast gene expression occurs only when expression of TWIST genes disappears in osteoblast precursors (29). TWIST proteins' antiosteogenic function is mediated by a TWIST box, which interacts with the RUNX2 DNA-binding domain to inhibit its function.

Transcriptional activity of RUNX2 might also be regulated by $1 \alpha, 25$-dihydroxy vitamin D3 through $V D R$. 
It was reported previously that RUNX2 and VDR are co-expressed in developing skeletal elements, and interaction between VDR and RUNX2 stabilises transcriptional complexes at specific promoters and induces osteoblast-specific genes expression such as osteocalcin (Ocn) (30).

In our study, both groups of subjects had low serum vitamin D concentration (below target range: $30-80 \mathrm{ng} /$ $\mathrm{mL}$ ), and there were no statistically significant differences between studied groups. However, a decrease in VDR gene expression was observed in the group with insulin resistance in comparison to the control group, which could in consequence affect bone remodelling. Our data showed that osteocalcin gene expression was not regulated, which could be explained by the fact that osteocalcin is a late marker of osteoblasts differentiation (31). However, the expression of some markers of cell differentiation towards osteoblasts as osteopontin, bone sialoprotein and collagen were upregulated in subjects with insulin resistance, which may confirm the presence of osteoprogenitors in the circulation.

Obese subjects with insulin resistance contrary to subjects without insulin resistance, responded to glucose load with a suppressed decrease in serum bone formation markers: total and carboxylated osteocalcin. Such data are in agreement with recently published results indicating that in obese subjects the decrease in serum cOC was suppressed in comparison to non-obese volunteers after oral glucose load $(32,33)$. Taking into consideration the fact that cOC accumulates mainly in bone (only 15\% is released into circulation) its decrease during OGTT in blood of subjects without insulin resistance could suggest increased bone formation. However, we did not observe suppression of ucOC decrease in obese subjects with insulin resistance in comparison to the control group as was reported in the study by Schwetz (34). On the other hand, Pollock et al. reported that in humans, cOC may influence insulin sensitivity, whereas ucOC could affect $\beta$-cell function and seems to be important in subjects with pre-diabetes and diabetes (35).

We postulate that after nutrients ingestion an increase in inflammatory response and insulin resistance could contribute to bone turnover alterations. Although we did not observe increases in pro-inflammatory cytokines on protein levels in serum at fasting, expression of genes taking part in inflammatory response that is CXCL8, CXCL10, CXCL9, CXCL11 was upregulated in subjects with insulin resistance in comparison to subjects without insulin resistance. Such gene expression patterns could influence osteoblasts differentiation. In vitro studies reported that pro-inflammatory cytokines such as tumour necrosis factor $\alpha$ (TNFA) suppressed osteoblast maturation by inhibiting the expression of RUNX2 (36). Pro-inflammatory cytokines disrupt the WNT signalling pathway. Resolution of inflammation was accompanied by striking changes in the expression of regulators of the Wnt/ $\beta$-catenin pathway (37).

Data from previous studies revealed that proinflammatory cytokines can stimulate osteoclastogenesis by modulating the RANK/RANKL/OPG system (27). Binding RANKL to its receptor, RANK, on the surface of osteoclasts and their precursors regulates the differentiation of precursors into multinuclear osteoclasts and osteoclast activation. Osteoprotegerin (OPG, TNFSF11) is secreted by osteoblasts and osteogenic stromal stem cells and protects the skeleton from excessive bone resorption by binding to RANKL and preventing it from interacting with RANK. The RANKL/OPG ratio is therefore a determinant of bone mass. Indeed, we found increased expression of TNFSF11, TNFRSF11B and RANK in obese subjects with insulin resistance. However, expression ratio TNFSF11/TNRSF11B was equal to 1 , which suggests that osteoclastogenesis is not activated in these subjects. Moreover, PU.1 (SPI1) and the master regulator of osteoclast differentiation nuclear factor of activated T-cells cytoplasmic 1 (NFATC1), which regulate a number of osteoclast-specific genes, were downregulated in obese subjects with insulin resistance (38). We also observed that expression of osteoclast-specific genes including ACP5 (TRAP), ITGA5 was downregulated. These results suggest that insulin resistance in obesity leads to inhibition of osteoblastogenesis but does not stimulate osteoclastogenesis and bone resorption.

Analysis of miRNA expression in whole blood of obese subjects with insulin resistance appears to confirm gene expression patterns. MicroRNAs (miRs/miRNAs) are endogenous small, non-coding RNAs that positively or negatively regulate gene expression and cellular processes via the RNA interference pathway $(39,40)$. By targeting mRNA transcripts post-translationally, they provoke either translational repression or degradation.

Analysis of miR expression revealed decreased expression of miR-29b, which has been shown to induce osteoblast differentiation. In vitro studies showed that miR-29b stimulates osteoblastogenesis by inhibiting protein expression of negative regulators of the cellular signal cascade transcription programmes, including for example TGFB3 (transforming growth factor beta) and DUSP2 (dual specificity protein phosphatase 2) (20). Mir-29b also stimulates osteogenic differentiation of unrestricted somatic stem cells from human cord 
blood (41). We found not only downregulation of miR 29b but also upregulation of TGFB3 and DUSP 2 mRNA.

Inhibition of miR-181a expression in subjects with insulin resistance in obesity also indicates bone defects. Recent studies presented overexpression of miR-181a in monocytes of lean persons. Moreover, downregulation of miR-181a expression was associated with a higher number of metabolic syndrome components $(42,43)$.

Transfection of sense miR-210-stimulated osteoblastic differentiation of murine stromal BM-MSC ST2 cells by positive regulation of the activin $A$ receptor type-1B (AcvR1b), which inhibits TGF- $\beta$ /activin signalling pathway (44). However, miR-210 expression was inhibited in whole blood from obese subjects with insulin resistance.

We also observed downregulation of miR-324-3p, which could suggest bone formation defects in subjects with insulin resistance. Recent studies presented that circulating miR-324-3p levels correlated with areal bone mineral density at the lumbar spine (18). Another study revealed positive association of miR-324-3p with bone microstructure and histomorphometry (19).

Summing up, results of our studies suggest defects in osteoblastogenesis in subjects with insulin resistance via mRNA downregulation for key signalling molecules in osteoblastogenesis, increased mRNA expression of osteoblastogenesis antagonists and inhibition of miRNA expression participating in regulation of osteoblasts differentiation. Defects in bone remodelling are reflected on protein levels by suppression of serum cOC decrease during OGTT in obese subjects with insulin resistance in comparison to individuals without insulin resistance. However, our study had some limitations. Firstly, the number of subjects included in the study was small and the number of both sexes was unequal. Secondly, mRNA and miRNA gene expression patterns due to financial limitations were analysed in a smaller group of subjects than biochemical parameters. However, the participants subjected to molecular tests reflected, in relation to the results of biochemical measurements, the whole two groups of subjects in the study (data not shown). One of the limitations of the study was also the fact that during OGTT we measured one marker of bone remodelling - osteocalcin, which is a non-collagenous protein produced by osteoblasts. This protein is considered a late marker of bone formation and increases at late stages of mineralisation. To reflect bone turnover other markers of bone formation (products of collagen synthesis (N-terminal propeptide of type I procollagen, PINP) and bone-specific alkaline phosphatase (BSAP)) as well as bone resorption (C-terminal telopeptide of type I collagen (CTX)) markers should be measured. Last but not least, subjects included in the study did not have bone microstructure analysed. It could be interesting to confirm that inhibition of osteoblastogenesis gene and $\mathrm{miR}$ expression in whole blood of obese subjects with insulin resistance indeed reflect bone microstructure defects. However, we believe that our results could contribute to expand knowledge about circulating components of bone turnover and could be a basis for further studies evaluating bone remodelling defects in different disease states.

Declaration of interest

The authors declare that there is no conflict of interest that could be perceived as prejudicing the impartiality of the research reported.

\section{Funding}

This work was supported European Commission through its Seventh Framework Programme "BIOmarkers of Robustness of Metabolic Homeostasis for Nutrigenomics-derived Health CLAIMS Made on Food" (BIOCLAIMS) (grant agreement number 244995); the National Science Centre (Poland, grant number DEC -2011/02/A/NZ2/00022) and Polish Ministry of Science and Higher Education grant number (K/ZDS/006287)

\section{Acknowledgements}

The authors thank Monika Chojnacka for technical support in methodology as well as assistance in language and text editing.

\section{References}

1 Ferron M, Wei J, Yoshizawa T, Del Fattore A, DePinho RA, Teti A, Ducy P \& Karsenty G. Insulin signaling in osteoblasts integrates bone remodeling and energy metabolism. Cell 2010142 296-308. (https:// doi.org/10.1016/j.cell.2010.06.003)

2 Hauschka PV, Lian JB, Cole DE \& Gundberg CM. Osteocalcin and matrix gla protein: vitamin K-dependent proteins in bone. Physiological Reviews 198969 990-1047. (https://doi.org/10.1152/ physrev.1989.69.3.990)

3 Price PA, Rice JS \& Williamson MK. Conserved phosphorylation of serines in the Ser-X-Glu/Ser(P) sequences of the vitamin K-dependent matrix gla protein from shark, lamb, rat, cow, and human. Protein Science 19943 822-830. (https://doi.org/10.1002/pro.5560030511)

4 Lee NK, Sowa H, Hinoi E, Ferron M, Ahn JD, Confavreux C, Dacquin R, Mee PJ, McKee MD, Jung DY, et al. Endocrine regulation of energy metabolism by the skeleton. Cell $2007 \mathbf{1 3 0} 456-469$. (https://doi.org/10.1016/j.cell.2007.05.047)

5 Ferron M \& Lacombe J. Regulation of energy metabolism by the skeleton: osteocalcin and beyond. Archives of Biochemistry and Biophysics 2014561 137-146. (https://doi.org/10.1016/j. abb.2014.05.022)

6 Ferron M, Hinoi E, Karsenty G \& Ducy P. Osteocalcin differentially regulates beta cell and adipocyte gene expression and affects the development of metabolic diseases in wild-type mice. Proceedings of the National Academy of Sciences of the United States of America 2008 105 5266-5270. (https://doi.org/10.1073/pnas.0711119105)

7 Razny U, Fedak D, Kiec-Wilk B, Goralska J, Gruca A, Zdzienicka A, Kiec-Klimczak M, Solnica B, Hubalewska-Dydejczyk A \& MalczewskaMalec M. Carboxylated and undercarboxylated osteocalcin in 
metabolic complications of human obesity and prediabetes. Diabetes/ Metabolism Research and Reviews 201733 e2862. (https://doi. org/10.1002/dmrr.2862)

8 Lin X, Brennan-Speranza TC, Levinger I \& Yeap BB. Undercarboxylated osteocalcin: experimental and human evidence for a role in glucose homeostasis and muscle regulation of insulin sensitivity. Nutrients 201810 847. (https://doi.org/10.3390/ nu10070847)

9 Marupanthorn K, Tantrawatpan Ch, Kheolamai P, Tantikanlayaporn D \& Manochantr S. Bone morphogenetic protein-2 enhances the osteogenic differentiation capacity of mesenchymal stromal cells derived from human bone marrow and umbilical cord. International Journal of Molecular Medicine 201739 654-662. (https:// doi.org/10.3892/ijmm.2017.2872)

10 Eghbali-Fatourechi GZ, Mödder UI, Charatcharoenwitthaya N, Sanyal A, Undale AH, Clowes JA, Tarara JE \& Khosla S. Characterization of circulating osteoblast lineage cells in humans. Bone 200740 1370-1377. (https://doi.org/10.1016/j. bone.2006.12.064)

11 Muto A, Mizoguchi T, Udagawa N, Ito S, Kawahara I, Abiko Y, Arai A, Harada S, Kobayashi Y, Nakamichi Y, et al. Lineage-committed osteoclast precursors circulate in blood and settle down into bone. Journal of Bone and Mineral Research 201126 2978-2990. (https://doi. org/10.1002/jbmr.490)

12 Zhou Y, Deng HW \& Shen H. Circulating monocytes: an appropriate model for bone-related study. Osteoporosis International 201526 2561-2572. (https://doi.org/10.1007/s00198-015-3250-7)

13 Matsuzaki K, Udagawa N, Takahashi N, Yamaguchi K, Yasuda H, Shima N, Morinaga T, Toyama Y, Yabe Y, Higashio K, et al. Osteoclast differentiation factor (ODF) induces osteoclast-like cell formation in human peripheral blood mononuclear cell cultures. Biochemical and Biophysical Research Communications 1998246 199-204. (https://doi. org/10.1006/bbrc.1998.8586)

14 Shalhoub V, Elliott G, Chiu L, Manoukian R, Kelley M, Hawkins N, Davy E, Shimamoto G, Beck J, Kaufman SA, et al. Characterization of osteoclast precursors in human blood. British Journal of Haematology 2000111 501-512. (https://doi.org/10.1046/j.13652141.2000.02379.x)

15 Mizoguchi T, Muto A, Udagawa N, Arai A, Yamashita T, Hosoya A, Ninomiya T, Nakamura H, Yamamoto Y, Kinugawa S, et al. Identification of cell cycle-arrested quiescent osteoclast precursors in vivo. The Journal of Cell Biology 2009184 541-554. (https://doi. org/10.1083/jcb.200806139)

16 Mari A, Pacini G, Murphy E, Ludvik B \& Nolan JJ. A model based method for assessing insulin sensitivity from the oral glucose tolerance test. Diabetes Care 200124 539-548. (https://doi. org/10.2337/diacare.24.3.539)

17 Matsuda M \& DeFronzo RA. Insulin sensitivity indices obtained from oral glucose tolerance testing: comparison with the euglycemic insulin clamp. Diabetes Care 199922 1462-1470. (https://doi. org/10.2337/diacare.22.9.1462)

18 Feichtinger X, Muschitz C, Heimel P, Baierl A, Fahrleitner-Pammer A, Redl H, Resch H, Geiger E, Skalicky S, Dormann R, et al. Bone-related circulating microRNAs miR-29b-3p, miR-550a-3p, and miR-324-3p and their association to bone microstructure and histomorphometry. Scientific Reports 20188 4867. (https://doi.org/10.1038/s41598-01822844-2)

19 Kocijan R, Muschitz C, Geiger E, Skalicky S, Baierl A, Dormann R, Plachel F, Feichtinger X, Heimel P, Fahrleitner-Pammer A, et al. Circulating microRNA signatures in patients with idiopathic and postmenopausal osteoporosis and fragility fractures. Journal of Clinical Endocrinology and Metabolism 2016101 4125-4134. (https:// doi.org/10.1210/jc.2016-2365)

20 Li Z, Hassan MQ, Jafferji M, Aqeilan RI, Garzon R, Croce CM, van Wijnen AJ, Stein JL, Stein GS \& Lian JB. Biological functions of miR$29 \mathrm{~b}$ contribute to positive regulation of osteoblast differentiation.
The Journal of Biological Chemistry 2009284 15676-15684. (https:// doi.org/10.1074/jbc.M809787200)

21 Viljakainen H, Ivaska KK, Paldánius P, Lipsanen-Nyman M, Saukkonen T, Pietiläinen KH, Andersson S, Laitinen K \& Mäkitie O. Suppressed bone turnover in obesity: a link to energy metabolism? A case-control study. Journal of Clinical Endocrinology and Metabolism 201499 2155-2163. (https://doi.org/10.1210/ jc.2013-3097)

22 Semenov M, Tamai K \& He X. SOST is a ligand for LRP5/LRP6 and a Wnt signaling inhibitor. Journal of Biological Chemistry 2005280 26770-26775. (https://doi.org/10.1074/jbc.M504308200)

23 Mao B, Wu W, Davidson G, Marhold J, Li M, Mechler BM, Delius H, Hoppe D, Stannek P,Walter C, et al. Kremen proteins are Dickkopf receptors that regulate Wnt/beta-catenin signaling. Nature 2002417 664-667. (https://doi.org/10.1038/nature756)

24 Mao B, Wu W, Li Y, Hoppe D, Stannek P, Glinka A \& Niehrs C. LDLreceptor-related protein 6 is a receptor for Dickkopf proteins. Nature 2001411 321-325. (https://doi.org/10.1038/35077108)

25 Peng Y, Kang Q, Cheng H, Li X, Sun MH, Jiang W, Luu HH, Park JY, Haydon RC \& He TC. Transcriptional characterization of bone morphogenetic proteins (BMPs)-mediated osteogenic signaling. Journal of Cellular Biochemistry 200390 1149-1165. (https://doi. org/10.1002/jcb.10744)

26 Tan X, Weng T, Zhang J, Wang J, Li W, Wan H, Lan Y, Cheng X, Hou N, Liu H, et al. Smad4 is required for maintaining normal murine postnatal bone homeostasis. Journal of Cell Science $2007 \mathbf{1 2 0}$ 2162-2170. (https://doi.org/10.1242/jcs.03466)

27 Asagiri M \& Takayanagi $\mathrm{H}$. The molecular understanding of osteoclast differentiation. Bone 200740 251-264. (https://doi. org/10.1016/j.bone.2006.09.023)

28 Kronenberg HM. Twist genes regulate Runx2 and bone formation. Developmental Cell 20046 317-318. (https://doi.org/10.1016/S15345807(04)00069-3)

29 Bialek P, Kern B, Yang X, Schrock M, Sosic D, Hong N, Wu H, Yu K, Ornitz DM, Olson EN, et al. A twist code determines the onset of osteoblast differentiation. Developmental Cell $20046423-435$ (https://doi.org/10.1016/S1534-5807(04)00058-9)

30 Paredes R, Arriagada G, Cruzat F, Villagra A, Olate J, Zaidi K, van Wijnen A, Lian JB, Stein GS, Stein JL, et al. Bone-specific transcription factor Runx2 interacts with the $1 \alpha, 25$-dihydroxyvitamin D3 receptor to up-regulate rat osteocalcin gene expression in osteoblastic cells. Molecular \& Cellular Biology 200424 8847-8861. (https://doi. org/10.1128/MCВ.24.20.8847-8861.2004)

31 Huang W, Yang S, Shao J \& Li YP. Signaling and transcriptional regulation in osteoblast commitment and differentiation. Frontiers in Bioscience: A Journal and Virtual Library 200712 3068-3092. (https:// doi.org/10.2741/2296)

32 Bjarnason NH, Henriksen EE, Alexandersen P, Christgau S, Henriksen DB \& Christiansen C. Mechanism of circadian variation in bone resorption. Bone 200230 307-313. (https://doi.org/10.1016/ s8756-3282(01)00662-7)

33 Paldanius PM, Ivaska KK, Hovi P, Andersson S, Eriksson JG, Väänänen K, Kajantie E \& Mäkitie O. Total and carboxylated osteocalcin associate with insulin levels in young adults born with normal or very low birth weight. PLOS ONE 20138 e63036. (https:// doi.org/10.1371/journal.pone.0063036)

34 Schwetz V, Lerchbaum E, Schweighofer N, Hacker N, Trummer O, Borel O, Pieber TR, Chapurlat R \& Obermayer-Pietsch B. Osteocalcin levels on oral glucose load in women being investigated for polycystic ovary syndrome. Endocrine Practice 201420 5-14. (https:// doi.org/10.4158/EP13110.OR)

35 Pollock NK, Bernard PJ, Gower BA, Gundberg CM, Wenger K, Misra S, Bassali RW \& Davis CL. Lower uncarboxylated osteocalcin concentrations in children with prediabetes is associated with betacell function. Journal of Clinical Endocrinology and Metabolism 201196 E1092-E1099. (https://doi.org/10.1210/jc.2010-2731) 
36 Gilbert L, He X, Farmer P, Rubin J, Drissi H, van Wijnen AJ, Lian JB, Stein GS \& Nanes MS. Expression of the osteoblast differentiation factor RUNX2 (Cbfa1/AML3/Pebp2 $\alpha$ A) is inhibited by tumor necrosis factor- $\alpha$. Journal of Biological Chemistry 2002277 2695-2701. (https://doi.org/10.1074/jbc.M106339200)

37 Matzelle MM, Gallant MA, Condon KW, Walsh NC, Manning CA, Stein GS, Lian JB, Burr DB \& Gravallese EM. Resolution of inflammation induces osteoblast function and regulates the Wnt signaling pathway. Arthritis and Rheumatism 201264 1540-1550. (https://doi.org/10.1002/art.33504)

38 Takayanagi H. Osteoimmunology: shared mechanisms and crosstalk between the immune and bone systems. Nature Reviews. Immunology 20077 292-304. (https://doi.org/10.1038/nri2062)

39 Lian JB, Stein GS, van Wijnen AJ, Stein JL, Hassan MQ, Gaur T \& Zhang Y. MicroRNA control of bone formation and homeostasis. Nature Reviews. Endocrinology 20128 212-227. (https://doi. org/10.1038/nrendo.2011.234)

40 Hassan MQ, Tye CE, Stein GS \& Lian JB. Non-coding RNAs: epigenetic regulators of bone development and homeostasis. Bone 201581 746-756. (https://doi.org/10.1016/j.bone.2015.05.026)
41 Trompeter HI, Dreesen J, Hermann E, Iwaniuk KM, Hafner M, Renwick N, Tuschl T \& Wernet P. MicroRNAs miR-26a, miR-26b, and miR-29b accelerate osteogenic differentiation of unrestrictedsomatic stem cells from human cord blood. BMC Genomics 201314111. (https://doi.org/10.1186/1471-2164-14-111)

42 Hulsmans M, Sinnaeve P, Van der Schueren B, Mathieu C, Janssens S $\&$ Holvoet P. Decreased miR-181a expression in monocytes of obese patients is associated with the occurrence of metabolic syndrome and coronary artery disease. Journal of Clinical Endocrinology and Metabolism 201297 E1213-E1218. (https://doi.org/10.1210/jc.20121008)

43 Zheng H, Liu J, Tycksen E, Nunley R \& McAlinden A. MicroRNA181a/b-1 over-expression enhances osteogenesis by modulating PTEN/PI3K/AKT signaling and mitochondrial metabolism. Bone 2019 123 92-102. (https://doi.org/10.1016/j.bone.2019.03.020)

44 Mizuno Y, Tokuzawa Y, Ninomiya Y, Yagi K, Yatsuka-Kanesaki Y, Suda T, Fukuda T, Katagiri T, Kondoh Y, Amemiya T, et al. miR-210 promotes osteoblastic differentiation through inhibition of AcvR1b. FEBS Letters 2009583 2263-2268. (https://doi.org/10.1016/j. febslet.2009.06.006)

Received 15 July 2019

Revised version received 10 September 2019

Accepted 17 September 2019 